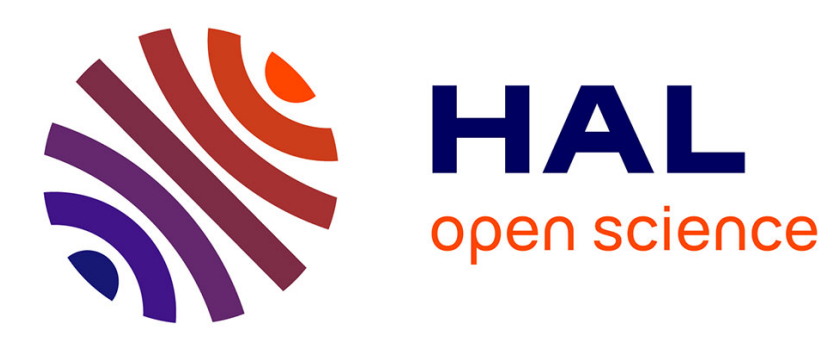

\title{
Ultrasonic propagation of reflected waves in cancellous bone: Application of Biot theory.
}

M Sadouki, M Fellah, Z.E.A Fellah, Erick Ogam, Claude L. Depollier

\section{To cite this version:}

M Sadouki, M Fellah, Z.E.A Fellah, Erick Ogam, Claude L. Depollier. Ultrasonic propagation of reflected waves in cancellous bone: Application of Biot theory.. 6th European Symposium on Ultrasonic Characterization of Bone (ESUCB 2015), Jun 2015, Corfou, Greece. 10.1109/ESUCB.2015.7169900 . hal-01309349

\section{HAL Id: hal-01309349 \\ https://hal.science/hal-01309349}

Submitted on 29 Apr 2016

HAL is a multi-disciplinary open access archive for the deposit and dissemination of scientific research documents, whether they are published or not. The documents may come from teaching and research institutions in France or abroad, or from public or private research centers.
L'archive ouverte pluridisciplinaire HAL, est destinée au dépôt et à la diffusion de documents scientifiques de niveau recherche, publiés ou non, émanant des établissements d'enseignement et de recherche français ou étrangers, des laboratoires publics ou privés. 


\section{Ultrasonic propagation of reflected waves in cancellous bone: Application of Biot theory}

Conference Paper · June 2015

DOI: 10.1109/ESUCB.2015.7169900

READS

44

5 authors, including:

\section{Mohamed Fellah}

University of Science and Technology Houa..

126 PUBLICATIONS 750 CITATIONS

SEE PROFILE

\section{Erick Ogam}

French National Centre for Scientific Resea. .

74 PUBLICATIONS 370 CITATIONS

SEE PROFILE

\section{Zea Fellah}

Laboratoire de Mécanique et d'Acoustique 120 PUBLICATIONS 1,208 CITATIONS

SEE PROFILE

\section{Claude Depollier}

Université du Maine

179 PUBLICATIONS 1,919 CITATIONS

SEE PROFILE 


\section{Ultrasonic propagation of reflected waves in cancellous bone: Application of Biot theory.}

\section{Fellah}

Laboratoire de Physique Théorique, Faculté de Physique, USTHB,

BP 32 El Alia, Bab Ezzouar 16111, Algeria

\author{
M. Sadouki \\ Faculté des Sciences et de la Technologie, Université Djilali \\ Bounaama à Khemis Miliana \\ BP 44225, Ain Defla, Algérie.
}

\author{
Z.E.A. Fellah and E.Ogam \\ LMA, CNRS, UPR 7051, Aix-Marseille Univ, Centrale \\ Marseille, \\ F-13402 Marseille Cedex 20, France.
}

\begin{abstract}
C. Depollier
LUNAM Université du Maine, UMR CNRS 6613

Laboratoire d'Acoustique de l'Université du Maine UFR STS, Avenue O. Messiaen, 72085 Le Mans CEDEX 09, France / National Research University "MPEI" Moscow, Russia.
\end{abstract}

\begin{abstract}
An ultrasonic propagation in cancellous bone is considered using the Biot theory modified by the Johnson et al. Numerical simulations of reflected waves in the time domain are worked out by varying the modified Biot parameters. The sensitivity of different mechanical parameters: Young modulus and the Poisson ratio of the skeletal frame and physical parameters, porosity, tortuosity and viscous characteristic length are studied showing their effect on the reflected ultrasonic waves of the bone sample. The sensitivity of the modified Biot parameters with respect to the reflected wave depends strongly on the coupling between the solid and fluid phases of the cancellous bone. We show from these simulations that some parameters such as porosity and tortuosity play an important role on reflected wave; the remaining parameters have low sensitivity compared with the porosity and tortuosity. Experimental results for reflected waves by human cancellous bone samples are given and compared with theoretical predictions.
\end{abstract}

Keywords-Ultrasonic; Reflected waves; Bone concellous

\section{INTRODUCTION}

Ultrasound can be used to characterize the elastic properties of cortical and cancellous bone. Fry and Barger [1] considered both types of bone when investigating the human skull while Ashman et al. [2] used ultrasound to measure the elastic properties of cancellous bone. Since trabecular bone is an inhomogeneous porous medium, the interaction between ultrasound and bone will be highly complex. Modeling ultrasonic propagation through trabecular tissue has been considered using porous media theories, such as Biot's theory $[3,4,6]$. The Biot model treats both individual and coupled behavior of the frame and pore fluid. Energy loss is considered to be caused by the viscosity of the pore fluid as it moves relative to the frame. The model predicts that sound velocity and attenuation in a two phase media will depend on frequency[7,8], the elastic properties of the constituting materials, porosity, permeability, tortuosity, and effective stress. This method should allow us to relate the physical parameters of our phantom to ultrasonic velocity and attenuation.
The purpose of this article is to study the ultrasonic reflected waves by a cancellous bone samples using Biot's theory modified by Johnson et al.[10]. Numerical simulations of reflective waves in the time domain of the bone sample are worked out by means of a variation in the parameters of a porous medium and the sensitivity of each parameter is studied. Experimental results for reflected waves through samples of human cancellous bone are given and a comparison with theoretical predictions is made

\section{MODEL}

The equations of motion of the frame and fluid are given by the Euler equations applied to the Lagrangian density. Here $\vec{u}$ and $\vec{U}$ are the displacements of the solid and fluid phases. The equations of motion are $[9,10]$

$$
\begin{gathered}
\rho_{11} \frac{\partial^{2} \vec{u}}{\partial t^{2}}+\rho_{12} \frac{\partial^{2} \vec{U}}{\partial t^{2}}=P \nabla(\nabla \cdot \vec{u})+Q \nabla(\nabla \cdot \vec{U})-N \nabla \wedge(\nabla \wedge \vec{u}) \\
\rho_{12} \frac{\partial^{2} \vec{u}}{\partial t^{2}}+\rho_{22} \frac{\partial^{2} \vec{U}}{\partial t^{2}}=Q \nabla(\nabla \cdot \vec{u})+R \nabla(\nabla \cdot \vec{U}) .
\end{gathered}
$$

wherein $\rho_{i j} \quad(\mathrm{i}=1,2$ and $\mathrm{j}=1,2)$ are the "mass coefficients" which are related to the densities of solid $\rho_{s}$ and fluid $\rho_{f}$ phases by $\rho_{11}=(1-\phi) \rho_{s}-\rho_{12}$, and $\rho_{22}=\phi \rho_{f}-\rho_{12}$, The coefficient $\rho_{12}$ represents the mass coupling parameter between the fluid and solid phases and is always negative $\rho_{12}=-\rho_{f}(\alpha(\omega)-1), \alpha(\omega)$ function of frequency, called the dynamic tortuosity $[10,11,12$,$] . To express the viscous$ exchanges between the fluid and the structure which play an important role in damping the acoustic wave in porous material. The parts of the fluid affected by this exchange can be estimated by the ratio of a microscopic characteristic length of the medium, for example pore size, to the viscous skin depth thickness $\delta=\sqrt{2 \eta / \omega \rho_{f}}$ ( $\eta$ : fluid viscosity, $\omega$ : angular frequency). At high frequencies, the viscous skin thickness is very thin near the radius of the pore $r$. The viscous effects are concentrated in a small volume near the surface of the frame 
$\delta / r \ll 1$. In this case, the expression of the dynamic tortuosity $\alpha(\omega)$ is given by[11]

$$
\alpha(\omega)=\alpha_{\infty}\left(1+\frac{2}{\Lambda}\left(\frac{\eta}{j \omega \rho_{f}}\right)^{1 / 2}\right)
$$

wherein $\alpha_{\infty}$ is the tortuosity and $\Lambda$ is the viscous characteristic length[11]. $P, Q$, and $R$ are generalized elastic constants which are related to other, measurable quantities, namely $\varphi$ (porosity), $K f$ (bulk modulus of the pore fluid), $K s$ (bulk modulus of the elastic solid), and $K b$ (bulk modulus of the porous skeletal frame). $N$ is the shear modulus of the composite as well as that of the skeletal frame. The equations which relate $P, Q$, and $R$ to $\varphi, K f, K s, K b$, and $N$ are given by

$$
\begin{gathered}
P=\frac{(1-\varphi)\left(1-\varphi-\frac{K_{b}}{K_{S}}\right) K_{S}+\phi \frac{K_{S}}{K_{f}} K_{b}}{\left(1-\frac{K_{b}}{K_{S}}\right)-\varphi\left(1-\frac{K_{S}}{K_{f}}\right)}, \quad Q=\frac{\left(1-\varphi-\frac{K_{b}}{K_{S}}\right) \phi K_{S}}{\left(1-\frac{K_{b}}{K_{S}}\right)-\varphi\left(1-\frac{K_{S}}{K_{f}}\right)} . \\
R=\frac{\varphi^{2} K_{S}}{\left(1-\frac{K_{b}}{K_{S}}\right)-\varphi\left(1-\frac{K_{S}}{K_{f}}\right)}
\end{gathered}
$$

The Young modulus and the Poisson ratio of the solid Es, vs and of the skeletal frame $E b, v b$ depend on the generalized elastic constant $P, Q$, and $R$ via the relations

$$
K_{s}=\frac{E_{S}}{3\left(1-2 v_{s}\right)}, \quad K_{b}=\frac{E_{b}}{3\left(1-2 v_{b}\right)} \text { and } \quad N=\frac{E_{b}}{2\left(1+2 v_{b}\right)} \text {. }
$$

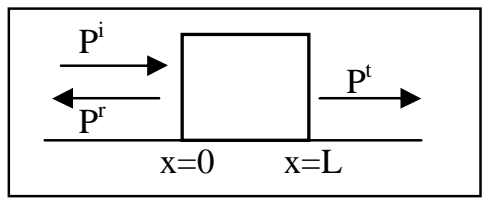

Fig 1. Geometry of the problem

For a slab of cancellous bone occupying the region $0 \leq x \leq L$ (Fig. 1), the general expression of reflected coefficient $\mathcal{R}(\omega)$ is given by $[5,10]$ :

with,

$$
\mathcal{R}(\omega)=\frac{F_{4}^{2}-1-F_{3}^{2}}{F_{3}^{2}-\left(1-F_{4}\right)^{2}}
$$

$$
\begin{gathered}
F_{4}=\sum_{\substack{i \\
k_{i} \psi_{i}}} F_{i} h_{i}, \quad F_{3}=\sum_{i} F_{i}, \\
F_{i}=\frac{\left.k\left(1-\mu_{i}\right)\right) \rho_{f} s^{2}, \quad i=1,2}{k \psi h_{i}}(1-\phi(1,2)
\end{gathered}
$$

In these equations, $\mathrm{k}$ is the propagation constant in the medium (I) and (III) with $\mathrm{k}=\omega / \mathrm{c}_{0}\left(\mathrm{c}_{0}\right.$ is the sound propagation velocity in these areas (I) and (III)).

$k_{i}$ are the constants of propagation of the two waves fast and slow, where:

$$
k_{i}^{2}=\frac{\omega^{2}}{2\left(P R-Q^{2}\right)}\left[\left(P \rho_{22}+R \rho_{11}-2 Q \rho_{12}\right)+(-1)^{i} \sqrt{\Delta}\right],
$$

with,

$$
\Delta=\left(P \rho_{22}+R \rho_{11}-2 Q \rho_{12}\right)^{2}-4\left(P R-Q^{2}\right)\left(\rho_{11} \rho_{22}-\rho_{12}^{2}\right)
$$

$\mu 1$ and $\mu 2$ are the ratios of amplitudes of the displacements for each of these waves fast and slow given by

$$
\mu_{1}=\frac{\omega^{2} \rho_{11}-P k_{1}^{2}}{\omega^{2} \rho_{12}-Q k_{1}^{2}}, \quad \quad \mu_{2}=\frac{\omega^{2} \rho_{11}-P k_{2}^{2}}{\omega^{2} \rho_{12}-Q k_{2}^{2}}
$$

and,

$$
\begin{gathered}
\psi_{1}=\varphi z_{2}-(1-\varphi) z_{4}, \quad \psi_{2}=(1-\varphi) z_{3}-\varphi z_{1} \\
\Psi=2\left(z_{1} z_{4}-z_{2} z_{3}\right)
\end{gathered}
$$

with,

$$
\begin{array}{ll}
z_{1}=\left(P+\mu_{1} Q\right) k_{1}^{2}, & z_{2}=\left(P+\mu_{2} Q\right) k_{2}^{2}, \\
z_{3}=\left(Q+\mu_{1} R\right) k_{1}^{2}, & z_{4}=\left(Q+\mu_{2} R\right) k_{2}^{2}
\end{array}
$$

The incident $p^{i}(t)$ and reflected $p^{r}(t)$ fields are related in time domain by the reflection scattering operator $R(t)$,

$$
\begin{aligned}
p^{r}(x, t) & =\int_{0}^{t} R(\tau) p^{i}\left(t-\tau+\frac{x}{c_{0}}\right) d \tau \\
& =R(t) * p^{i}(t) * \delta\left(t+\frac{x}{c_{0}}\right)
\end{aligned}
$$

In (13) $\mathrm{c}_{0}$ is the velocity outside the porous material, $*$ denotes the time convolution operation, and $\delta(t)$ is the delta function. The temporal operator kernel $R(t)$ is independent of the incident field used in the scattering experiment, its expression is calculated by taking the inverse Fourier transform of the reflection coefficient (6) for slab of cancellous bone. The scattering operator $R(t)$ is then calculated numerically from $\mathcal{R}(\omega)$ and its convolution with the incident signal gives us the reflected signal in the time domain.

\section{SENSITIVITY OF PHYSICAL AND MECANICAL PARAMETERS ON THE REFLECTED WAVES}

Numerical simulations of reflected waves (13) are run by varying the parameters of a cancellous bone described acoustically using the modified Biot's theory. The variation is applied to the governing parameters and is between $\pm 10 \%$ and $\pm 20 \%$. The numerical values chosen for the physical parameters are those taken from sample M1 (table 1.). The incident signal is given in figure 3 .

Among the important parameter that appears in theory of sound propagation in porous materials is porosity. Porosity $\varphi$ is the relative fraction, by volume, of the fluid contained within the material. According to the figure 2(a), a strong influence of variation of porosity on the reflected signal is observed. For a $10 \%$ increase of this parameter, the amplitude decreases to about $70 \%$, and for a decrease of $10 \%$, the amplitude increases by approximately $78.6 \%$. Therefore influence of the porosity $\varphi$ is marked over the entire waveform. So, porosity plays a very important role in the reflected signal.

Another important parameter in describing ultrasonic propagation in a porous medium is the tortuosity $\alpha_{\infty}$. The tortuosity expresses the sinuosity and change in diameter of the pores. The reader can see the sensitivity of tortuosity in reflected mode for a $\pm 10 \%$ change. An important change occurs at the amplitudes of the wave. From figure 2(b), a $10 \%$ increase tortuosity produces an $80.10 \%$ increase in the 
amplitude of the reflected wave and a decrease of $10 \%$ produces an attenuation of about $24.9 \%$ on the amplitude of reflected wave. It is deduced that the effect of tortuosity is also important on the reflected waves.

Figure 2(c) shows the sensitivity of viscous characteristic length $\Lambda$ introduced by Johnson et al.[11] to describe viscous exchanges between fluid and structure. According to this figure, we see that this parameter has little influence on the reflected signal, we note that for a variation of $50 \%$, we get an increase of $3.10 \%$ of the amplitude of the wave reflected, and as the numerical simulation shows, $\Lambda$ plays a less important role in reflection than does porosity $\varphi$ and tortuosity $\alpha_{\infty}$.

Figures 2(d), (e), (f) and (g) show a comparison between simulated reflected signals corresponding to mechanical parameters; the Young modulus and the Poisson ratio of the solid Es, vs and of the skeletal frame Eb, vb. No major changes were observed in wave amplitude. In this case the sensitivity of mechanical parameters is not very important for a $\pm 20 \%$ change in amplitude of reflected waves.

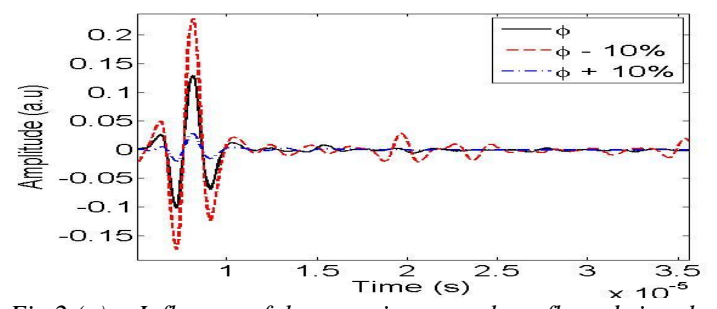

Fig.2 (a) - Influence of the porosity $\varphi$ on the reflected signal

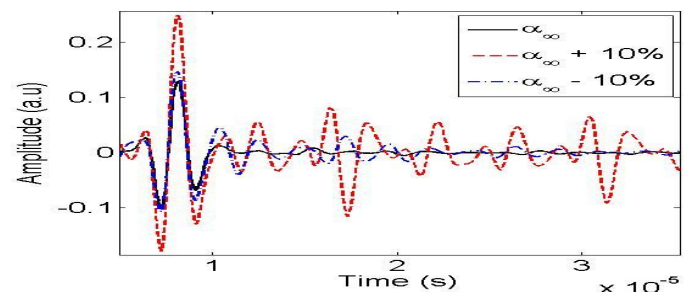

Fig.2 (b) - Influence of the tortuosity $\alpha_{\infty}$ on the reflected signal

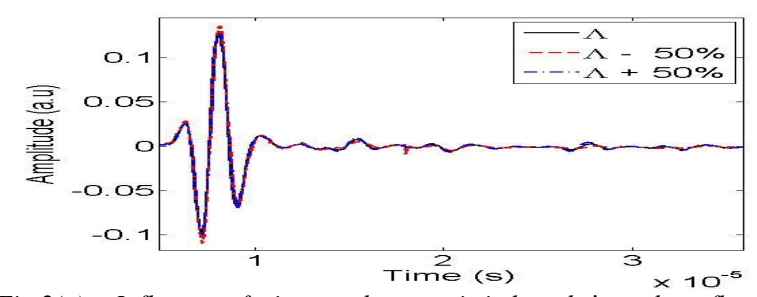

Fig.2(c) - Influence of viscous characteristic length $\Lambda$ on the reflected signal

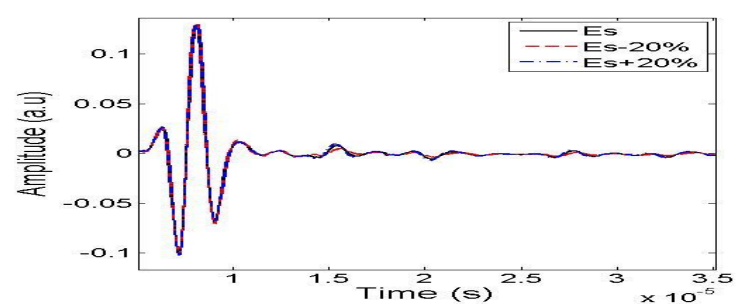

Fig.2(d) - Influence of Young modulus of the solid $E_{s}$ on the reflected signal

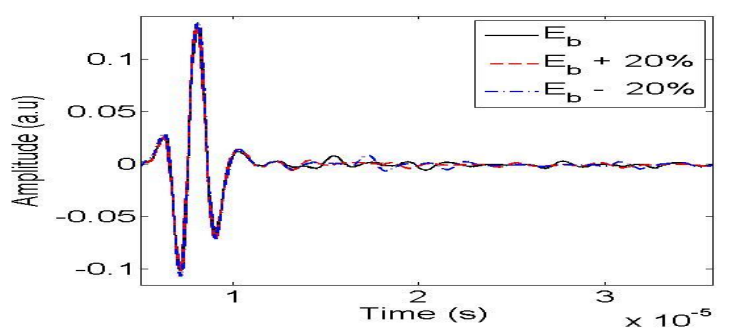

Fig.2(e) - Influence of Young modulus of the frame $E_{b}$ on the reflected signal

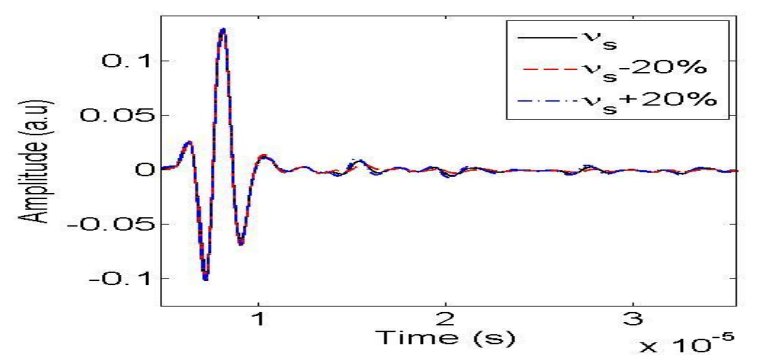

Fig. 2(f) - Influence of the Poison ratio of the solid $\nu_{\mathrm{s}}$ on the reflected signal

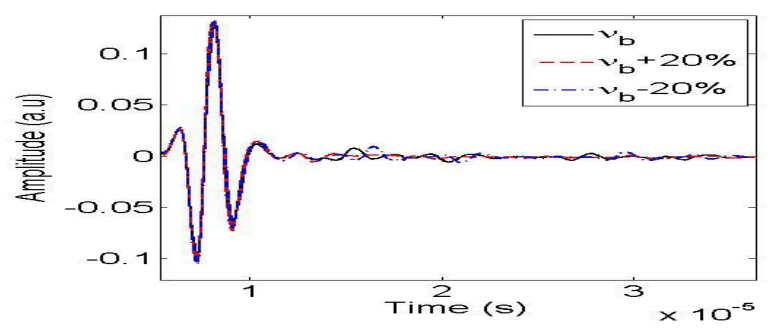

Fig. 2(g) - Influence of the ratio modulus of the fluid $\nu_{\mathrm{b}}$ on the reflected signal

As an application of this model, some numerical simulations are compared with experimental results. Experiments are performed in water using two broadband Panametrics A 306S piezoelectric transducers with a central frequency of 2.25 $\mathrm{MHz}$ in water. $400 \mathrm{~V}$ pulses are provided by a 5058PR Panametrics pulser/receiver. The signals received are amplified to $90 \mathrm{~dB}$ and filtered above $10 \mathrm{MHz}$ to avoid high frequency noise (energy is totally filtered by the sample in this upper frequency domain). Electronic interference is removed by 1000 acquisition averages. The parallel-faced cubic samples M1 and M2 of thickness L1 $=0.7 \mathrm{~cm}$ and $\mathrm{L} 2=0.9 \mathrm{~cm}$ were machined from human cancellous bone in femoral heads. Samples characteristics are measured using standard Methods $[11,12]$ and given in Table I. Fig. 3 shows the incident and reflected signals (at left) (pulse wave form traveling in water), and their spectrums (at right) of the two samples M1 and M2.

Figures 4(a) and (b) show the comparison between experimental reflected signal (solid line) and simulated signal (dashed line) given by (13) for both bone samples M1 and M2. The experimental data and theoretical prediction are seen to match closely, which allowed us to conclude that the modified Biot theory using the Johnson et al. model is quite suitable for 
describing the propagation of ultrasonic wave in cancellous bone.

TABLE1. VALUES OF THE PHYSICAL AND MECHANICLA PARAMETERS OF THE SAMPLE M1 AND M2

\begin{tabular}{|c|c|c|c|c|c|c|c|}
\hline Sample & $\varphi$ & $\alpha_{\infty}$ & $\Lambda(\mu \mathbf{m})$ & $\mathbf{E}_{\mathrm{s}}(\mathrm{GPa})$ & $\mathbf{E}_{\mathrm{b}}(\mathbf{G P a})$ & $v_{s}, v_{b}$ & $\rho_{\mathrm{s}}\left(\mathrm{kg} \cdot \mathrm{m}^{-3}\right)$ \\
\hline M1 & 0.9 & 1.03 & 90.0 & 20.0 & 1.1 & 0.3 & 1990 \\
\hline M2 & 0.9 & 1.01 & 15.5 & 20.0 & 8.5 & 0.3 & 1990 \\
\hline Fluid & \multicolumn{2}{|c|}{$P_{f}\left(K_{g} \cdot m^{-3}\right)$} & \multicolumn{2}{|c|}{$\mathbf{K}_{\mathbf{f}}(\mathbf{G P a})$} & \multicolumn{2}{|c|}{$\eta$ (Pas) } & \\
\hline & \multicolumn{2}{|c|}{1000} & \multicolumn{2}{|c|}{2.3} & \multicolumn{2}{|c|}{0.001} & \\
\hline
\end{tabular}
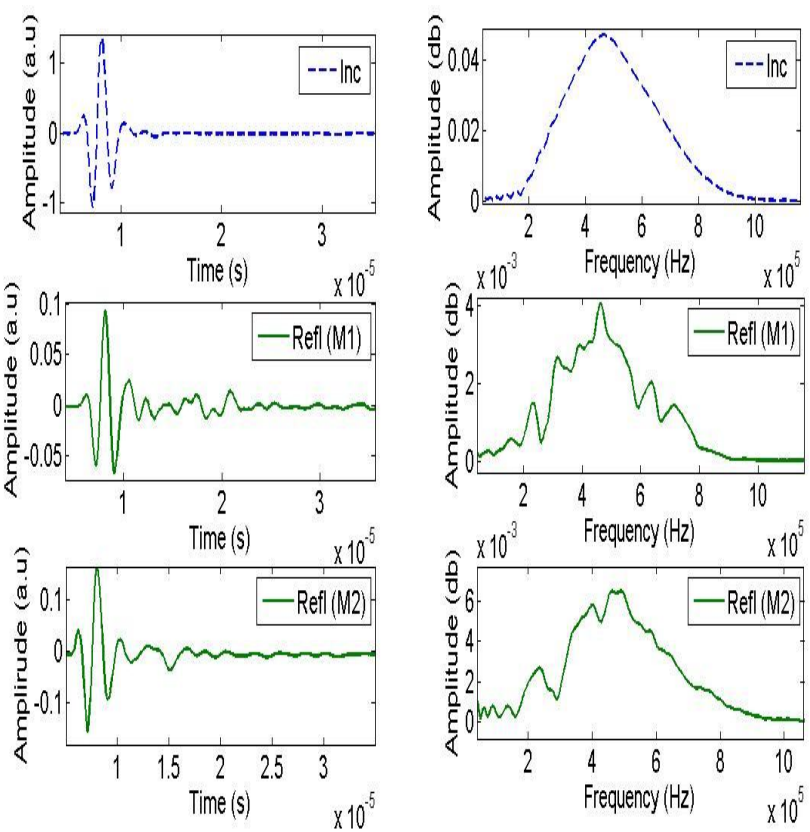

Fig. 3 - Incident (dashed blue line) and reflected waves (solid green line) of the sample bone M1 and M2 (at right) and their spectrums (at left).

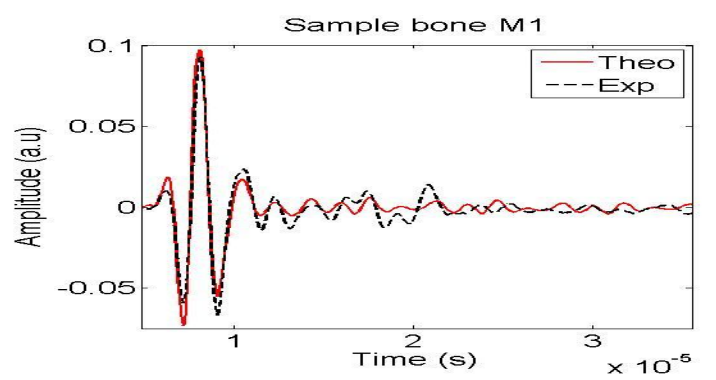

Fig. 4(a) - Comparison between experimental reflected signal (dashed line) and simulated signal (solid line)

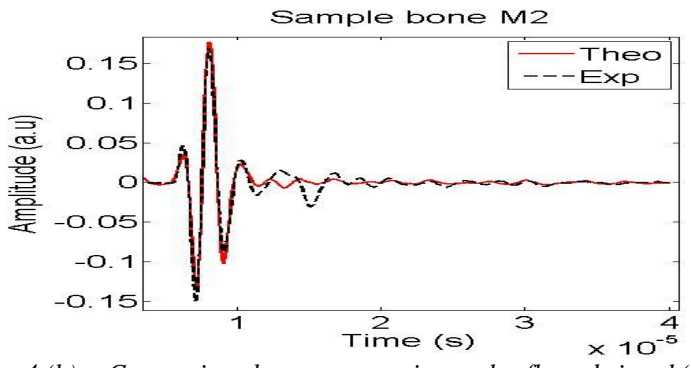

Fig. 4 (b) - Comparison between experimental reflected signal (dashed line) and simulated signal (solid line)

\section{CONCLUSION}

In this paper, experimental validation of Biot's theory modified by the Johnson et al. using reflected waves by samples of human cancellous bone was performed. Numerical simulations of reflected waves in the time domain were run by varying the parameters of a porous medium. The variation applied to the governing parameters was between $\pm 10 \%$ and $\pm 20 \%$, the sensitivity of each parameter was studied, showing the importance of the values of these parameters in reflected wave forms. Finally, experimental validation of this model using reflected waves by samples of human cancellous bone was performed and found to produce excellent agreement between theory and experiment. This leads to the conclusion that the expressions of scattering operators obtained are correct. One future hope is to solve the inverse problem and return to the physical parameters, porosity and tortuosity of the medium, from reflected experimental data.

\section{ACKNOWLEDGMENT}

C. Depollier is supported by the Russian Science Foundation under grant 14-49-00079.

\section{REFERENCES}

[1] F. J. Fry and J. E. Barger, "Acoustical properties of the humain skull," J. Acoust. Soc. Am. 63, 1576-1590 (1978).

[2] R. B. Ashman, J. D. Corin, and C. H. Turner, "Elastic properties of cancellous bone: Measurement by an ultrasonic technique,' J.Biomech. 10, pp 979-989 (1987).

[3] M. A. Biot, "The theory of propagation of elastic waves in fluidsaturated porous solid. I. Low frequency range,' J. Acoust. Soc. Am. 28, pp 168-178 (1956).

[4] M. A. Biot, "The theory of propagation of elastic waves in fluidsaturated porous solid. I. Higher frequency range,' J. Acoust. Soc. Am. 28, pp 179- 191 (1956).

[5] M. Fellah, Z. E. A. Fellah, F.G. Mitri, E. Ogam, C. Depollier, "Transient ultrasound propagation in porous media using Biot theory and fractional calculus: Application to human cancellous bone ," J. Acoust. Soc. Am. 133(4) pp 1867-1881 (2014).

[6] M. Pakula, F. Padilla, P. Laugier, and M. Kaczmarek, "Application of Biot's theory to ultrasonic characterization of human cancellous bones: Determination of structural, material, and mechanical properties," J. Acoust. Soc. Am. 123, pp 2415-2423 (2008).

[7] J. L. Buchanan, R. P. Gilbert, and M. J. Ou, "Transfer functions for a onedimensional fluid-poroelastic system subject to an ultrasonic pulse,"Nonlinear Anal.: Real World Appl. 13, pp 1030-1043 (2012).

[8] J. L. Buchanan, R. P. Gilbert, and M. Y. Ou, "Recovery of the parameters of cancellous bone by inversion of effective velocities, and transmission and reflection coefficients," Inverse Probl. 27, 125006 (2011).

[9] M. A. Biot, "The theory of propagation of elastic waves in fluidsaturated porous solid. I. Higher frequency range," J. Acoust. Soc. Am. 28, pp 179-191 (1956).

[10] Z. E. A. Fellah, J. Y. Chapelon, S. Berger, W. Lauriks, and C. Depollier, "Ultrasonic wave propagation in human cancellous bone: Application of Biot theory," J. Acoust. Soc. Am. 116 (1), pp 61-73 (2004).

[11] D. L. Johnson, J. Koplik, and R. Dashen, "Theory of dynamic permeability and tortuosity in fluid-saturated porous media,' J. Fluid Mech. 176, pp 379-402 (1987).

[12] J. F. Allard, Propagation of Sound in Porous Media: Modeling Sound Absorbing Materials Chapman and Hall, London, (1993). 